\title{
The Critical Role of Sleep Spindles in Hippocampal- Dependent Memory: A Pharmacology Study
}

\author{
Sara C. Mednick, ${ }^{1,2,3}$ Elizabeth A. McDevitt, ${ }^{1}$ James K. Walsh, ${ }^{4,5}$ Erin Wamsley, ${ }^{6}$ Martin Paulus, ${ }^{2,3}$ Jennifer C. Kanady, \\ and Sean P. A. Drummond ${ }^{2,3}$ \\ 'Department of Psychology, University of California Riverside, Riverside, California 92521, ${ }^{2}$ Department of Psychiatry, University of California San Diego, \\ La Jolla, California 92093, ${ }^{3}$ VA San Diego Healthcare System, La Jolla, California 92161, ${ }^{4}$ St. Luke’s Hospital, St. Louis, Missouri 64111, 5Department of \\ Psychiatry, Stanford University, Palo Alto, California 94305, ${ }^{6}$ Department of Psychiatry, Harvard Medical School, Boston, Massachusetts 02115, and \\ ${ }^{7}$ Department of Psychology, University of California Berkeley, Berkeley, California 94720
}

An important function of sleep is the consolidation of memories, and features of sleep, such as rapid eye movement (REM) or sleep spindles, have been shown to correlate with improvements in discrete memory domains. Because of the methodological difficulties in modulating sleep, however, a causal link between specific sleep features and human memory consolidation is lacking. Here, we experimentally manipulated specific sleep features during a daytime nap via direct pharmacological intervention. Using zolpidem (Ambien), a short-acting $\mathrm{GABA}_{\mathrm{A}}$ agonist hypnotic, we show increased sleep spindle density and decreased REM sleep compared with placebo and sodium oxybate (Xyrem). Naps with increased spindles produced significantly better verbal memory and significantly worse perceptual learning but did not affect motor learning. The experimental spindles were similar to control spindles in amplitude and frequency, suggesting that the experimental intervention enhanced normal sleep processes. Furthermore, using statistical methods, we demonstrate for the first time a critical role of spindles in human hippocampal memory performance. The gains in memory consolidation exceed sleep-alone or control conditions and demonstrate the potential for targeted, exceptional memory enhancement in healthy adults with pharmacologically modified sleep.

\section{Introduction}

Correlational studies of the effect of sleep on human memory indicate a role of defined sleep features on specific memory domains (Diekelmann and Born, 2010). For example, the number of sleep spindles, an electrophysiological marker of non-rapid eye movement (NREM) sleep, increases with hippocampaldependent learning (Eschenko et al., 2006), is temporally coupled with hippocampal sharp wave ripples after spatial exploration in rodents (Siapas and Wilson, 1998), facilitates the integration of newly learned information with existing knowledge (Tamminen et al., 2010), and correlates with better retention of hippocampaldependent verbal memories in humans (Gais et al., 2002; Schabus et al., 2004; Clemens et al., 2005; Schmidt et al., 2006). In contrast, in rodents after avoidance learning, (1) REM sleep increases, (2) memory is impaired without posttraining REM sleep, and (3) learning is associated with increased theta $(6-10 \mathrm{~Hz})$ activity during posttraining REM episodes (Fogel et al., 2009), which

\footnotetext{
Received July 2, 2012; revised Jan. 10, 2013; accepted Jan. 16, 2013.

Author contributions: S.C.M., J.K.W., M.P., and S.P.A.D. designed research;S.C.M., E.A.M., and J.C.K. performed research; S.C.M., E.A.M., and E.W. analyzed data; S.C.M., E.A.M., J.K.W., E.W., M.P., J.C.K., and S.P.A.D. wrote the paper.

This work was supported by NIMH Grant K01MH080992 (S.C.M.) and National Science Foundation Award 0729021 (S.P.A.D.). J.K.W. was supported by Pfizer Merck \& Co., Somnus, Vanda, Neurogen, sanofi-aventis, Ventus, Respironics, Apnex, and Jazz Pharmaceuticals. Jazz Pharmaceuticals provided the sodium oxybate.

J.K.W. has provided consulting services to Pfizer, sanofi-aventis, Respironics, Transcept, Neurogen, GlaxoSmithKline, Eli Lilly, Merck, Kingsdown, Vanda, Ventus, Vivus, and Somnus.

Correspondence should be addressed to Sara C. Mednick at the above address. E-mail: smednick@ucr.edu. DOI:10.1523/JNEUROSCI.3127-12.2013

Copyright $\odot 2013$ the authors $\quad 0270-6474 / 13 / 334494-11 \$ 15.00 / 0$
}

seems to be a recapitulation of encoding activity, or replay, during theta activity (Louie and Wilson, 2001). In humans, both perceptual learning (Mednick et al., 2003) and implicit priming (Cai et al., 2009) do not rely on the hippocampus and are facilitated by REM sleep.

Recently, important advances have been made by using experimental methods that manipulate sleep features to test their effects on memory, including transcranial stimulation to enhance (Marshall et al., 2006) or inhibit (Marshall et al., 2011) slow wave activity. However, interventional studies that examine the relationship between sleep spindles and human memory consolidation are lacking. Here we used a pharmacological approach to examine whether experimentally enhancing spindle activity could increase consolidation of hippocampal-dependent memories beyond that associated with naturally occurring spindles.

We compared two hypnotics, zolpidem (ZOL; Ambien; sanofi-aventis) and sodium oxybate (SO; Xyrem; Jazz Pharmaceuticals), which have been shown to increase (Brunner et al., 1991; Feinberg et al., 2000) and decrease (Walsh et al., 2010) spindle activity, respectively. ZOL is also associated with increased hippocampal sharp wave ripple complexes (Koniaris et al., 2011) and decreased and/or delayed REM sleep (Brunner et al., 1991; Feinberg et al., 2000), whereas SO is associated with increased slow wave sleep (SWS) and delta power in morning naps (Walsh et al., 2010). Tasks involving consolidation in three memory domains were chosen: the word-pair associates task (verbal), the texture discrimination task (TDT; perceptual), and the motor sequence task (motor). Improvement on the word- 
pair associates task has been shown to correlate with the number and density of sleep spindles (Gais et al., 2002; Schabus et al., 2004; Clemens et al., 2005), whereas improvement on the motor sequence task correlates with Stage 2 sleep (Walker et al., 2002) and fast-frequency spindle density (Nishida and Walker, 2007; Barakat et al., 2011). In contrast, perceptual learning on the texture discrimination task seems to depend on the presence of postlearning REM sleep (Stickgold et al., 1998; Mednick et al., 2003). Therefore, we hypothesized that pharmacological increases in sleep spindles with ZOL would benefit verbal and motor memory compared with placebo and SO, whereas experimental decreases in REM would produce a decrement in perceptual learning, compared with controls.

\section{Materials and Methods}

Subjects. A total of 49 participants (Study 1: $n=19,11$ females; Study 2: $n=30 ; 15$ females) between the ages of 18 and 39 ( $22 \pm 3$ years) who were normal sleepers and habitually obtained $\sim 8 \mathrm{~h}$ of sleep each night gave informed consent to participate in the experiment, which was approved by the Institutional Review Board of the University of California, San Diego.

Drugs. ZOL is a positive allosteric modulator at $\mathrm{GABA}_{\mathrm{A}}$ receptors with a short half-life $(1.5-4.5 \mathrm{~h})$, and rapid onset (mean $T_{\max }=1.6 \mathrm{~h}$ ) (Drover, 2004; Farrant and Nusser, 2005) that (1) enhances sleep spindle density assessed both visually and by spectral power density in the sigma range (12-15 Hz), (2) decreases and/or delays REM sleep (Brunner et al., 1991; Feinberg et al., 2000), and (3) increases hippocampal sharp wave ripple complexes (Koniaris et al., 2011). SO is a GABA agonist acting via $\mathrm{GABA}_{\mathrm{B}}$ receptors and although it also binds to $\gamma$-hydroxybutarate receptors, this pharmacologic property is not thought to contribute to the effects on sleep (Kaupmann et al., 2003; Andriamampandry et al., 2007). SO has a half-life of 30-60 min and reaches peak plasma level at 45-120 $\mathrm{min}$. SO decreases power density in the sigma range, and increases SWS and delta power in morning naps (Walsh et al., 2010).

General procedure. Subjects wore an actigraph and completed daily sleep diaries for the full period of the study to document sleep/wake schedules. Subjects were included in the study if their regular sleep schedule included going to bed between 10:00 P.M. and midnight and waking up between 6:00 and 8:00 A.M. During the study, subjects agreed to a $1 \mathrm{~h}$ bedtime window sometime between 10:00 P.M. and midnight and a $1 \mathrm{~h}$ wake time window sometime between 6:00 and 8:00 A.M. (corresponding as closely as possible to habitual sleep/wake schedules), with an average of $8 \mathrm{~h}$ of sleep per night. Sleep diaries and actigraphy documented participants' adherence to the agreed upon sleep/wake schedule for the duration of the study. Subjects were asked to refrain from alcohol, caffeine, and all stimulants for $48 \mathrm{~h}$ before and including the study day, since all three factors can affect sleep patterns during the overnight sleep portion and the ability to nap during day 2 . Starting the prohibition $48 \mathrm{~h}$ early also minimized the chance of active caffeine withdrawals during the study [although moderate-to-heavy caffeine users who are most likely to experience significant withdrawal symptoms were not enrolled (i.e., $>100 \mathrm{mg}$ per day)]. In Study 1 each participant had five drug conditions (10 mg of ZOL, $5 \mathrm{mg}$ of ZOL, $3 \mathrm{~g}$ of SO, $2.5 \mathrm{~g}$ of SO, placebo), and in Study 2, each participant had three drug conditions ( $10 \mathrm{mg}$ of ZOL, $2.5 \mathrm{~g} \mathrm{SO}$, placebo). Each study was separated by $5-10 \mathrm{~d}$ to allow for drug washout and recovery from any sleep changes related to the nap and/or study drugs. Both Study 1 and Study 2 were repeated-measures, crossover designs in which each subject experienced all drug conditions. Drug condition order was randomized.

After a night of sleep with polysomnography (PSG) monitoring, subjects were woken at 5:00 A.M. and given breakfast. Activities before the nap varied depending on the study. In Study 1, subjects were allowed to read and watch television. In Study 2, subjects were tested on the three cognitive tasks. At 8:30 A.M., subjects went back to their bed for an electrode check and nap. Drugs were administered immediately before lights out. This timing was chosen as the best method for establishing a uniform measure of sleep latency. The nap took place at 8:30 A.M. to

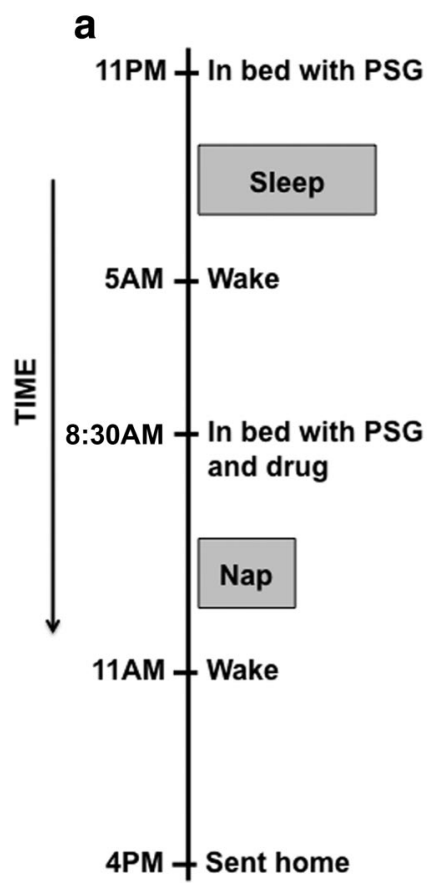

b

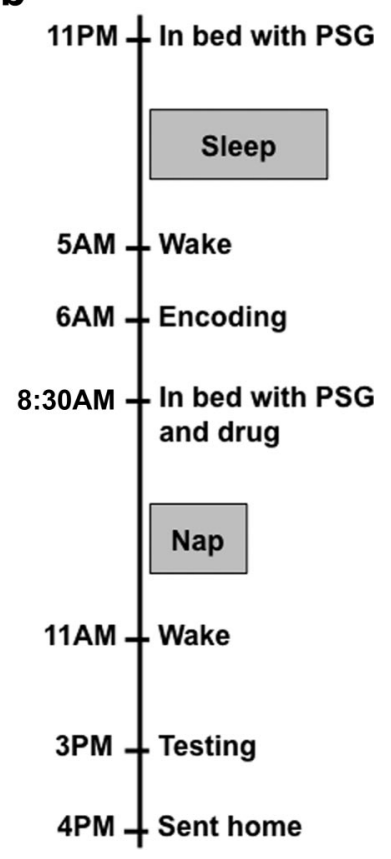

Figure 1. Study time line. The experimental time line for each session for Study 1 (a) and Study $2(\boldsymbol{b})$. On the eve of the experimental day, subjects slept in the laboratory with a bedtime of 11:00 P.M. and a wake time of 5:00 A.M. In study 2, participants took cognitive tests between 6:00 and 8:00 A.M. At 8:30 A.M., subjects were reattached to the PSG monitor, given their medication, and allowed to nap until 90 min of total sleep time was obtained. At 3:00 P.M., subjects were retested on the cognitive tasks.

capitalize on circadian fluctuations in REM sleep (highest in the morning). This allowed us to maximize differences in sleep stages between the drug and placebo conditions (Fig. 1a). Morning naps with placebo should be relatively rich in REM, whereas sleep spindles would be enhanced and REM diminished with ZOL, and SWS would be enhanced with SO compared with placebo. Subjects were allowed to sleep for up to $2 \mathrm{~h}$ of time in bed or until they achieved $90 \mathrm{~min}$ of sleep. Sleep was scored on-line to ensure that all subjects had the same total sleep time (Mednick et al., 2003). Study 1 allowed us to identify effects of drug and dose on specific sleep electroencephalographic (EEG) changes desired for the subsequent test of memory consolidation effects in Study 2 (Fig. 1b). Subjects were continuously monitored during the hours between the nap and the second test session (3:00 P.M.). They were allowed to watch television, eat lunch, shower, read, and work on the laboratory computer. Subjective sleepiness was measured five times during the day (6:00 A.M., 7:00 A.M., 10:30 A.M., 3:00 P.M., and 4:00 P.M.) using the Karolinska Sleepiness Scale (KSS).

Polysomnography. PSG included standard EEG, electro-oculographic (EOG), electromyographic (EMG), and electrocardiographic measures for recording sleep. On the first experimental night, nasal airflow, respiratory effort, blood oxygen concentration, and leg EMG were also recorded, to enable screening for sleep apnea and periodic limb movements. All PSG data were collected using Astro-Med Grass Heritage Model 15 amplifiers digitized at a sampling rate of $256 \mathrm{~Hz}$ using Grass Gamma software. Scalp EEG and EOG electrodes were referenced to unlinked opposite mastoids (C3/A2, O1/A2, C4/A1, O $2 / A 1$, LOC/A2, and ROC/A1), and muscle tone EMGs were attached under the chin. The high-pass filters were set at $0.3 \mathrm{~Hz}$ and the low-pass filters at $100 \mathrm{~Hz}$ for all EEGs and EOGs. A $60 \mathrm{~Hz}$ notch filter was also used to eliminate potential background noise. At the beginning of each recording, an internal $50 \mu \mathrm{V}$ calibration signal was generated followed by impedance checks and biocalibrations. Impedances for all electrodes were confirmed to be under 5 $\mathrm{k} \Omega$. Sleep stages were visually scored by two blind-to-condition raters in 30 s epochs according to Rechtschaffen and Kales criteria (Rechtschaffen and Kales, 1968). Sleep architecture variables included minutes and per- 
centage of Stage 1, Stage 2, SWS, and REM; also, total sleep time (TST), sleep latency (SL), and sleep efficiency (SE). EEG data were bandpass filtered between 0.3 and $35 \mathrm{~Hz}$, and all epochs with artifacts and arousals were manually rejected by visual inspection. We analyzed spindles during Stage 2 sleep, given our primary hypothesis of a relationship of Stage 2 sleep spindles with memory consolidation. Two blind-to-condition raters visually counted sleep spindles at both C3 (left hemisphere) and C4 (right hemisphere) electrodes, and Stage 2 spindle densities (spindles/ Stage 2 minutes) were calculated for C3 and C4.

Analysis. We examined actigraphy variables [TST ${ }^{\text {act }}, \mathrm{SL}^{\text {act }}, \mathrm{SE}^{\text {act }}$, and wake after sleep onset (WASO ${ }^{\text {act }}$ )] for the seven nights before the experimental day and sleep architecture (TST ${ }^{\text {night }}$, minutes of Stage $1^{\text {night }}$, Stage $2^{\text {night }}$, SWS $^{\text {night }}$, and REM ${ }^{\text {night }}$ ) on the eve of the experiment. Nap questionnaires, delivered after each nap, measured subjective sleepiness (KSS), subjective nap experience, drug effects, and mood. For these variables, repeated-measures ANOVA were used to test for differences across drug conditions. Variables for the experimental nap included sleep stages and spindle densities in Stage 2 sleep (i.e., Stage $1^{\text {nap }}$, Stage $2^{\text {nap }}$, SWS ${ }^{\text {nap }}$, $\mathrm{REM}^{\text {nap }}, \mathrm{TST}^{\text {nap }}, \mathrm{SL}^{\text {nap }}, \mathrm{SE}^{\text {nap }}$, spindle density of C3 and C4).

For statistical comparisons, we used a repeated-measures ANOVA and a priori $t$ tests to test differences in sleep and memory performance across drug conditions, Pearson correlations for comparing performance with spindle features (i.e., density, frequency, and amplitude), and an analysis of covariance (ANCOVA) to test whether the drug conditions had an effect on verbal memory performance after the variance due to spindle density was removed. We also used a contrast analysis to test the prediction that there would be a linear increase in verbal memory across conditions (i.e., $\mathrm{SO}<$ placebo $<\mathrm{ZOL})$. Contrast weights of $(-1,0,1)$ were compared with difference scores in verbal memory in SO, placebo, and ZOL, respectively.

Analysis of spindle features was conducted in BrainVision Analyzer 2.0, following Wamsley et al. (2012). Spindle amplitude and frequency were quantified based on analysis of $2 \mathrm{~s}$ EEG epochs centered on the point at which each spindle was manually marked. The amplitude of a spindle was defined as the maximal voltage in this $2 \mathrm{~s}$ window after $12-15 \mathrm{~Hz}$ bandpass filtering. The peak frequency of a spindle was defined as the frequency in the 11-16 Hz range showing maximal power spectral density (square microvolt per hertz) after fast Fourier transform (FFT) decomposition. Spindles with a peak frequency below $13.5 \mathrm{~Hz}$ were classified as "slow" spindles, whereas spindles with a peak frequency above $13.5 \mathrm{~Hz}$ were classified as "fast." For each subject, we computed average spindle density and amplitude, separately for fast and slow spindles (De Gennaro and Ferrara, 2003; Schabus et al., 2007; Wamsley et al., 2012). Spectral analysis of EEG activity examined the mean of total power (square microvolt) within the slow oscillation band $(0.5-1.0 \mathrm{~Hz})$ during NREM sleep. The power spectrum was calculated via FFT, using a Hamming window applied to all artifact-free $30 \mathrm{~s}$ epochs of NREM sleep (Stage 2 and SWS) during the night.

Cognitive tasks. In the word-pair associates task, subjects were visually presented 48 semantically related word pairs. Study words were twosyllable English words between four and seven letters in length and of moderate frequency (e.g., table-bench). Research assistants rated words on a 1-7 imagery scale as having imagery ratings between 5 and 7 . Word pairs (with the second word presented under the first to avoid lateralization effects) were presented on a computer screen placed at a distance of about $50 \mathrm{~cm}$ from the eyes. The letters were black on a white background and centered. Paired-associate lists of 144 words were arranged in six groups of 24 pairs, which were randomized across conditions. Subjects were presented with two word-pair lists in the morning session. Recall was cued (i.e., subjects were presented with the first word of each pair and had to recall the second one). Immediate recall was tested immediately after training, and subjects were shown the correct answer after each response. Delayed recall was tested during the postintervention testing session. Memory consolidation was measured as a differences score between the number of words recalled in the delayed recall test relative to the immediate recall test.

In the TDT, participants discriminated two targets per trial: a central letter ("T" or "L"), and a peripheral line array (vertical or horizontal orientation) in one of four quadrants (bottom left, top left, bottom right, and top right) at $2.5-5.9^{\circ}$ eccentricity from the center of the screen. The quadrant was randomized across conditions. The peripheral array consisted of three diagonal bars that were either positioned in a horizontal array or vertical array against a background of horizontally oriented distracters, which created a texture difference between the target and the background. An experimental trial consisted of the following sequence of four screens: central fixation cross, target screen for $32 \mathrm{~ms}$, blank screen for a duration between 0 and $600 \mathrm{~ms}$ [the interstimulus interval (ISI)], mask for $16 \mathrm{~ms}$, followed by the response time interval before the next trial. Subjects reported both the letter at central fixation (T or L) and the orientation of the peripheral, three-element array (horizontal or vertical) by making two key presses. The central task controlled for eye movements.

Each block consisted of 25 trials, each with the same ISI. A threshold was determined from the performance across 13 blocks, with a progressively shorter ISI, starting with $600 \mathrm{~ms}$ and ending with $0 \mathrm{~ms}$. The specific sequence of ISIs across an entire session was $[600,500,400,300,250,200$, $160,140,120,100,60,30,0]$. A psychometric function of percentage correct for each block was fit with a Weibull function to determine the ISI at which performance yielded $80 \%$ accuracy. TDT performance was calculated as the difference score of Session 1-Session 2 thresholds, so that a positive score indicates performance improvement (i.e., decreased ISI threshold in Session 2), whereas a negative score indicates perceptual deterioration (Mednick et al., 2002, 2005, 2008a,b).

Subjects practiced an easy version of the task (ISI of 1000-600 ms) on the day of their intake/physical examination. Practice consisted of five blocks with 25 trials within each block, with the peripheral target positioned in a quadrant that would not be trained during the study. This practice ensured that participants understood the task and were discriminating the peripheral target between $90 \%$ and $100 \%$ correct on the easiest version of the task.

The motor sequence task involved pressing four numeric keys using the fingers of the nondominant hand to repeat a five-element sequence "as quickly and accurately as possible" for a trial period of $30 \mathrm{~s}$, followed by 30 s of rest. Three motor patterns containing completely unique grammars (sequence $X, 4-1-3-2-4$; sequence $Y, 3-1-2-4-2$; sequence $Z$, 1-4-32-1) were available, and subjects were assigned one pattern to learn (sequences were randomized across conditions). The Encoding session consisted of 12 contiguous trials ( $12 \mathrm{~min}$ to complete), and the Test session consisted of a repetition of the same 12 contiguous trials from Session 1. Performance measures were the number of complete sequences achieved per trial ("speed") and the number of errors made relative to the number of correct sequences ("accuracy"). We computed a difference score between the average number of correct sequences for the last three blocks of the encoding session and the first three blocks of the test session.

Subjective drug effects. Subjective reports of drug effects, including decreased appetite, dizziness, dry mouth, elevated mood, faintness, headache, and so on, were significantly different across conditions ( $p=$ $0.012)$, with increased symptom reports for low SO $(p=0.07)$, high SO $(p=0.03)$, and high ZOL $(p=0.02)$ compared to placebo. Dosedependent increases in sadness were found $(p=0.01)$ for ZOL, compared with placebo. No differences were found in happiness, stress, relaxation, irritability, or anxiety. Subjective experience of the nap differed across conditions in the number of wake episodes $(p<0.001)$, where all conditions reported significantly fewer wake episodes than placebo $(p<0.01)$. Subjects felt they slept longer $(p=0.03)$ with both doses of SO compared with placebo. Sleepiness after waking measured by KSS was significantly different across conditions $(p=0.016)$, where subjects reported being significantly sleepier after low SO $(p=0.018)$ and high $\mathrm{ZOL}(p=0.015)$ compared with placebo. No differences, however, were found in subjective assessment of latency to fall asleep across drug conditions and subjective time awake after sleep onset.

Adverse events. In Study 1, 19 participants completed five drug studies each. Across 95 challenges, 33 instances of a minor adverse event were reported by participants. The five discrete events reported were drowsiness $(n=15)$, dizziness $(n=9)$, headache $(n=3)$, nausea $(n=5)$, and vomiting $(n=1)$. Thirty percent of the events were reported after low SO: drowsiness $(n=2)$, dizziness $(n=2)$, headache $(n=2)$, nausea $(n=$ 
Table 1. Nocturnal sleep: week prior (actigraphy)

\begin{tabular}{|c|c|c|c|c|c|c|}
\hline & Placebo & Sodium oxybate $(2.5 \mathrm{~g})$ & Sodium oxybate $(3 \mathrm{~g})$ & Zolpidem (5 mg) & Zolpidem (10 mg) & Statistic \\
\hline \multicolumn{7}{|l|}{ Study 1} \\
\hline $\mathrm{TST}^{\text {act }}(\mathrm{min})$ & $397 \pm 59$ & $396 \pm 52$ & $388 \pm 63$ & $413 \pm 56$ & $408 \pm 59$ & $F_{(4,44)}=0.70, p=0.60$ \\
\hline SE ${ }^{\text {act }}$ & $77 \pm 8$ & $78 \pm 8$ & $76 \pm 9$ & $80 \pm 7$ & $78 \pm 7$ & $F_{(4,44)}=0.68, p=0.62$ \\
\hline WASO $^{\text {act }}$ (min) & $98 \pm 43$ & $89 \pm 35$ & $96 \pm 36$ & $83 \pm 34$ & $91 \pm 36$ & $F_{(4,44)}=0.38, p=0.81$ \\
\hline \multicolumn{7}{|l|}{ Study 2} \\
\hline $\mathrm{TST}^{\text {act }}(\min )$ & $376 \pm 7.2$ & $381 \pm 6.5$ & & & $372 \pm 6.9$ & $F_{(2,54)}=0.80, p=0.45$ \\
\hline SE ${ }^{\text {act }}$ & $81 \pm 1.2$ & $81 \pm 1.0$ & & & $80 \pm 1.3$ & $F_{(2,54)}=1.14, p=0.32$ \\
\hline WASO $^{\text {act }}$ (min) & $71 \pm 4.6$ & $71 \pm 4.7$ & & & $75 \pm 4.9$ & $F_{(2,54)}=0.63, p=0.53$ \\
\hline
\end{tabular}

Table 2. Nocturnal sleep: experimental night (polysomnography)

\begin{tabular}{|c|c|c|c|c|c|c|}
\hline & Placebo & Sodium oxybate $(2.5 \mathrm{~g})$ & Sodium oxybate $(3 \mathrm{~g})$ & Zolpidem (5 mg) & Zolpidem (10 mg) & Statistic \\
\hline \multicolumn{7}{|l|}{ Study 1} \\
\hline $\mathrm{TST}^{\text {night }}$ (min) & $322 \pm 30$ & $313 \pm 44$ & $333 \pm 17$ & $321 \pm 29$ & $323 \pm 42$ & $F_{(4,72)}=1.38, p=0.28$ \\
\hline SE ${ }^{\text {night }}$ & $89 \pm 7$ & $86 \pm 12$ & $92 \pm 4$ & $89 \pm 8$ & $89 \pm 11$ & $F_{(4,72)}^{(1,12)}=1.06, p=0.41$ \\
\hline$S L^{\text {night }}$ (min) & $16 \pm 16$ & $18 \pm 26$ & $9 \pm 5$ & $12 \pm 15$ & $15 \pm 24$ & $F_{(4,72)}=1.00, p=0.43$ \\
\hline WASO $^{\text {night }}(\mathrm{min})$ & $20 \pm 17$ & $29 \pm 32$ & $18 \pm 11$ & $25 \pm 27$ & $21 \pm 27$ & $F_{(4,72)}=0.49, p=0.73$ \\
\hline Stage 1 (min) & $12 \pm 6$ & $13 \pm 7$ & $12 \pm 8$ & $12 \pm 5$ & $12 \pm 6$ & $F_{(4,72)}=0.23, p=0.92$ \\
\hline Stage 2 (min) & $175 \pm 21$ & $171 \pm 23$ & $183 \pm 24$ & $166 \pm 24$ & $172 \pm 34$ & $F_{(4,72)}=2.32, p=0.10$ \\
\hline SWS (min) & $77 \pm 26$ & $77 \pm 25$ & $80 \pm 24$ & $79 \pm 22$ & $81 \pm 28$ & $F_{(4,72)}=0.25, p=0.90$ \\
\hline REM (min) & $57 \pm 17$ & $50 \pm 19$ & $57 \pm 17$ & $62 \pm 18$ & $56 \pm 20$ & $F_{(4,72)}=2.07, p=0.13$ \\
\hline \multicolumn{7}{|l|}{ Study 2} \\
\hline $\mathrm{TST}^{\text {night }}(\mathrm{min})$ & $319 \pm 26$ & $317 \pm 30$ & & & $321 \pm 24$ & $F_{(2,58)}=0.46, p=0.62$ \\
\hline SE ${ }^{\text {night }}$ & $86 \pm 18$ & $88 \pm 8$ & & & $87 \pm 18$ & $F_{(2,58)}=0.19, p=0.82$ \\
\hline$S L^{\text {night }}$ (min) & $13 \pm 9$ & $18 \pm 14$ & & & $14 \pm 12$ & $F_{(2,58)}=0.46, p=0.62$ \\
\hline WASO $^{\text {night }}$ (min) & $23 \pm 21$ & $25 \pm 26$ & & & $23 \pm 18$ & $F_{(2,58)}=0.06, p=0.93$ \\
\hline Stage 1 (min) & $14 \pm 9$ & $15 \pm 10$ & & & $13 \pm 9$ & $F_{(2,58)}=0.71, p=0.49$ \\
\hline Stage 2 (min) & $190 \pm 34$ & $190 \pm 36$ & & & $192 \pm 31$ & $F_{(2,58)}=0.11, p=0.89$ \\
\hline SWS (min) & $71 \pm 22$ & $70 \pm 30$ & & & $71 \pm 25$ & $F_{(2,58)}=0.07, p=0.92$ \\
\hline REM (min) & $45 \pm 20$ & $42 \pm 14$ & & & $46 \pm 13$ & $F_{(2,58)}=0.53, p=0.59$ \\
\hline
\end{tabular}

$3)$, and vomiting $(n=1)$. Thirty percent of the events were reported after high SO: drowsiness $(n=4)$, dizziness $(n=4)$, headache $(n=1)$, nausea $(n=1)$, and vomiting $(n=0)$. Nine percent of the events were reported after low ZOL: drowsiness $(n=3)$, dizziness $(n=0)$, headache $(n=0)$, nausea $(n=0)$, and vomiting $(n=0)$. Thirty percent of the events were reported after high ZOL: drowsiness $(n=6)$, dizziness $(n=3)$, headache $(n=0)$, nausea $(n=1)$, and vomiting $(n=0)$. No adverse events were reported after placebo.

In Study 2, 30 participants completed three challenges each. Across 90 challenges, 42 instances of a minor adverse event were reported by participants. The five discrete events reported were drowsiness $(n=17)$, dizziness $(n=12)$, nausea $(n=4)$, vomiting $(n=2)$, and diarrhea $(n=$ $1)$. Thirty-six percent of the events were reported after SO: drowsiness $(n=5)$, dizziness $(n=7)$, nausea $(n=2)$, vomiting $(n=1)$, and diarrhea $(n=0)$. Fifty-five percent of the events were reported after ZOL: drowsiness $(n=11)$, dizziness $(n=8)$, nausea $(n=2)$, vomiting $(n=1)$, and diarrhea $(n=1)$. Nine percent of the events were reported after placebo: drowsiness $(n=3)$, dizziness $(n=1)$, nausea $(n=0)$, vomiting $(n=0)$, and diarrhea $(n=0)$.

All drug side effects had worn off before subjects left the laboratory in the afternoon. Additionally, all subjects were cleared as safe to leave alone by a research nurse. However, transportation was provided for all subjects regardless of condition.

\section{Results}

Study 1

Nocturnal sleep: week prior and experimental night

Subjects maintained a regular sleep/wake schedule for the duration of the study as can be seen from the actigraphy data (Table 1). Repeated-measures ANOVA on the averages of sleep variables (i.e., TST $^{\text {act }}, \mathrm{SE}^{\text {act }}$, and WASO ${ }^{\text {act }}$ ) from the week before each experimental night showed no significant differences, indicating that subjects adhered to sleep schedules across the 5 weeks of the study $\left(\mathrm{TST}^{\mathrm{act}}, p=0.60 ; \mathrm{SE}^{\mathrm{act}}, p=0.62\right.$; $\left.\mathrm{WASO}^{\mathrm{act}}, p=0.81\right)$. The PSG-recorded night of sleep indicated no differences in sleep parameters across the five conditions ( $2.5 \mathrm{~g}$ of SO, $3 \mathrm{~g}$ of SO, $5 \mathrm{mg}$ of ZOL, $10 \mathrm{mg}$ of ZOL, placebo) on the eve of the experimental day (Table 2) $\left(\mathrm{TST}^{\text {night }}, p=0.28\right.$; $\mathrm{SL}^{\text {night }}, p=0.43$; $\mathrm{SE}^{\text {night }}, p=$ 0.41 ; WASO ${ }^{\text {night }}, p=0.73$ ). By design, the experimental night had significantly less sleep compared with average sleep from the week prior (paired $t$ test; $2.5 \mathrm{~g}$ of SO, $p=0.001 ; 3 \mathrm{~g}$ of SO, $p=$ $0.001 ; 5 \mathrm{mg}$ of ZOL, $p<0.001 ; 10 \mathrm{mg}$ of ZOL, $p<0.001$; placebo, $p<0.001)$. The experimental night also had less WASO across all five conditions ( $2.5 \mathrm{~g}$ of SO, $p<0.001 ; 3 \mathrm{~g}$ of SO, $p<0.001 ; 5 \mathrm{mg}$ of ZOL, $p<0.001$; $10 \mathrm{mg}$ of ZOL, $p<0.001$; placebo, $p<0.001$ ). SL was shorter in the $3 \mathrm{~g} \mathrm{SO}(p<0.001)$ condition, but not in the other four conditions. Greater SE was found in all five experimental nights ( $2.5 \mathrm{~g}$ of SO, $p=0.04 ; 3 \mathrm{~g}$ of SO, $p<0.001 ; 5 \mathrm{mg}$ of $\mathrm{ZOL}, p=0.001 ; 10 \mathrm{mg}$ of ZOL, $p=0.002$; placebo, $p<0.001$ ). We analyzed differences in sleep stages across the five experimental nights and found no differences: TST ${ }^{\text {night }}(p=0.29)$, Stage $1^{\text {night }}(p=0.92)$, Stage $2^{\text {night }}(p=0.10)$, SWS ${ }^{\text {night }}(p=0.90)$, and $\operatorname{REM}^{\text {night }}(p=0.13)$.

\section{Experimental nap sleep characteristics}

To control for circadian confounds and avoid nocturnal sleep disruption, we used daytime sleep sessions at 8:30 A.M. This further allowed us to take advantage of the observation that 8:30 A.M. naps have high proportions of REM sleep. Analysis of sleep during the experimental naps showed that our paradigm produced the hypothesized differences in sleep spindle density and sleep stages (Fig. $2 a$ ). We compared sleep parameters across the experimental nap and found the drug conditions produced more consolidated sleep compared with placebo (Table 3). Although 
total sleep time (TST ${ }^{\text {nap }}$ ) did not significantly differ across five conditions $(p=$ $0.10)$, sleep latency (SL $\left.{ }^{\text {nap }}\right) \operatorname{did}(p=0.06)$, but no conditions were significantly different from placebo. Also, wake after sleep onset (WASO ${ }^{\text {nap }}$ ) significantly differed across conditions $(p=0.01)$, with decreased waking in $3 \mathrm{~g}$ of SO $(p=0.001)$ and $10 \mathrm{mg}$ of ZOL $(p=0.058)$ compared with placebo. Sleep efficiency ( $S E^{\text {nap }}$ ) was also significantly different $(p=0.006)$, with increased efficiency in $2.5 \mathrm{~g}$ of SO $(p=0.03), 3 \mathrm{~g}$ of $\mathrm{SO}(p=0.008)$, and 10 $\mathrm{mg}$ of ZOL $(p=0.049)$ compared with placebo.

Sleep spindle density in Stage 2 (C3, left hemisphere) was significantly different across conditions $(p<0.001)$, with enhanced spindle density in $10 \mathrm{mg}$ of ZOL $(p=0.002)$ and diminished spindle density in $2.5 \mathrm{~g}$ of SO $(p<0.001)$ compared with placebo. Similarly, spindle density in C4 (right hemisphere) showed the same pattern of results $(p<0.001)$. REM differed across conditions $(p=0.03)$ because of decreased REM in the $5 \mathrm{mg}$ ZOL condition compared with placebo $(p=$ $0.01)$. Also, SO produced more SWS compared with placebo $(p=0.003)$, and the post hoc test showed that all conditions were greater than placebo $(p=0.01)$. Minutes in Stage 2 were not different across conditions $(p<0.67)$. All four drug conditions produced less Stage 1 than in the placebo condition $(p<0.001)$. The finding that we could systematically alter sleep architecture to increase or decrease specific sleep features allowed us to study how these changes affect memory consolidation across three specific memory domains.

\section{Study 2: Role of pharmacologically increased spindles in memory \\ consolidation}

Our central experimental question was whether increases in spindle density affect memory consolidation. To this end, Study 2 ( $n=30)$ conditions were selected based on Study 1 dosageresponse findings with the addition of a memory encoding session at 6 A.M. and test of memory consolidation at 3 P.M. (Fig. $1 b$ ). We selected doses of SO and ZOL to maximize the predicted differences in spindle density while minimizing drug side effects (i.e., $10 \mathrm{mg}$ of ZOL enhanced spindle density, and $2.5 \mathrm{~g}$ of SO diminished spindle density in Study 1).

Nocturnal sleep: week prior and experimental night

Repeated-measures ANOVA on the averages of actigraph sleep variables (i.e., $\mathrm{TST}^{\text {act }}, \mathrm{SE}^{\text {act }}$, and $\mathrm{WASO}^{\text {act }}$ ) from the week before each experimental night showed no significant differences, indicating that subjects adhered to sleep schedules across the 5 weeks of the study (Table 1). We analyzed differences in sleep stages across the five experimental nights and found no differences (Table 2).

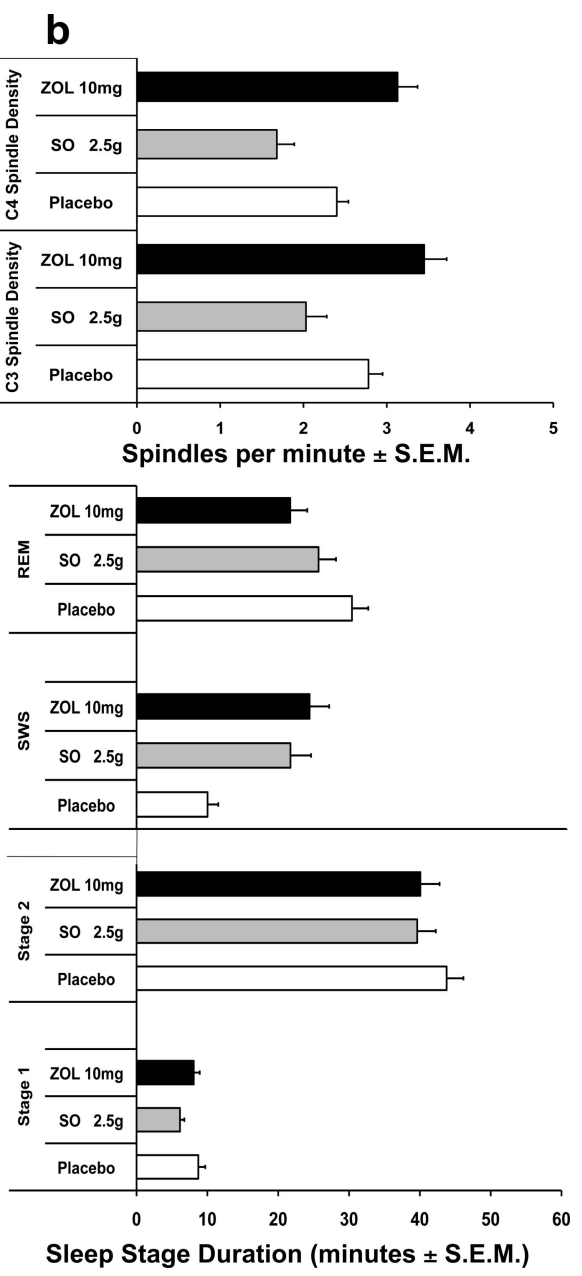

Sleep Stage Duration (minutes \pm S.E.M.)

Figure 2. Drug effects on minutes in each sleep stage and spindle density. $\boldsymbol{a}$, Study 1 compares 5 and $10 \mathrm{mg}$ of ZOL (black bars), 2.5 and $3 \mathrm{~g}$ of $\mathrm{SO}$ (gray bars), and placebo (white bars). b, Study 2 compares $10 \mathrm{mg}$ of $\mathrm{ZOL}$ (black bars), $2.5 \mathrm{~g}$ of SO (gray bars), and placebo (white bars). Statistical comparisons are between drug conditions and placebo. ${ }^{* * *} p<0.001$; ${ }^{* *} p<0.01 ;{ }^{*} p<0.05$.

\section{Pharmacologically modified sleep}

We replicated spindle density and sleep stage profiles from Study 1 across conditions (Fig. 2b, Table 4). REM differed across conditions $(p=0.02)$ due to decreased REM in the ZOL condition compared with placebo $(p=0.004)$. Also, SO $(p<0.001)$ and ZOL $(p<0.001)$ conditions had greater SWS than placebo. Minutes of Stage 2 sleep were not different across conditions $(p<$ $0.34)$. Stage 1 differed ( $p=0.002)$ with fewer minutes during SO compared with placebo $(p=0.001)$.

For Stage 2 spindle density, we examined hemispheric differences between C 3 and C4 using a $3 \times 2$ (drug condition $\times$ hemisphere) repeated-measures ANOVA and found no hemispheric differences in spindle density in the drug by hemisphere interaction $(p=0.72)$. For clarity, we present only C4 data for the drug/behavior interactions, as the outcome for C3 was comparable (Table 5). Importantly, sleep spindle density during Stage 2 sleep differed across conditions $(p=0.002)$, with increased density in ZOL $(p=0.002)$ and decreased density in SO $(p=0.002)$ compared with placebo.

For fast and slow Stage 2 spindle density and amplitude, hemispheric differences were examined using a $3 \times 2 \times 2$ (drug condition $\times$ hemisphere $\times$ fast vs slow spindles) repeated-measures ANOVA and found no hemispheric differences in density (threeway interaction, $p=0.487$ ) and amplitude (three-way interaction, $p=0.595$ ) across drug conditions. We also compared 
Table 3. Study 1: experimental nap (polysomnography)

\begin{tabular}{|c|c|c|c|c|c|c|}
\hline & Placebo & $\begin{array}{l}\text { Sodium } \\
\text { oxybate }(2.5 \mathrm{~g})\end{array}$ & $\begin{array}{l}\text { Sodium } \\
\text { oxybate }(3 \mathrm{~g})\end{array}$ & $\begin{array}{l}\text { Zolpidem } \\
(5 \mathrm{mg})\end{array}$ & $\begin{array}{l}\text { Zolpidem } \\
(10 \mathrm{mg})\end{array}$ & Statistic \\
\hline $\mathrm{TST}^{\text {nap }}$ (min) & $86 \pm 4.5$ & $91 \pm 1.7$ & $91 \pm 4.6$ & $85 \pm 2.5$ & $95 \pm 1.4$ & $F_{(4,72)}=2.00, p=0.10$, partialeta $^{2}=0.1$ \\
\hline Sleep latency ${ }^{\text {nap }}$ (min) & $11.7 \pm 2.0$ & $8.2 \pm 1.0$ & $9.0 \pm 2.1$ & $13.4 \pm 1.7$ & $10.6 \pm 1.5$ & $F_{(4,72)}=3.00, p=0.06$, partialeta $^{2}=0.11$ \\
\hline WASO $^{\text {nap }}(\mathrm{min})$ & $16.7 \pm 4.9$ & $10 \pm 5.0$ & $5.5 \pm 3.0$ & $14.6 \pm 4.8$ & $6.5 \pm 1.3$ & $F_{(4,71)}=3.26, p=0.01$, partialeta $^{2}=0.15$ \\
\hline Sleep efficiency ${ }^{\text {nap }}$ & $76 \pm 4.2$ & $85.4 \pm 2.9$ & $87.1 \pm 4.5$ & $77.4 \pm 3.7$ & $85.5 \pm 1.8$ & $F_{(4,72)}=3.97, p=0.006$, partialeta $^{2}=0.18$ \\
\hline C4 spindle density & $2.70 \pm 0.20$ & $1.32 \pm 0.20$ & $1.14 \pm 0.23$ & $3.12 \pm 0.39$ & $3.49 \pm 0.46$ & $F_{(4,72)}=24.84, p<0.001$, partialeta $^{2}=0.58$ \\
\hline C3 spindle density & $3.11 \pm 0.36$ & $1.71 \pm 0.25$ & $1.44 \pm 0.25$ & $3.55 \pm 0.40$ & $4.08 \pm 0.48$ & $F_{(4,72)}=19.99, p<0.001$, partialeta $^{2}=0.52$ \\
\hline Stage 1 (min) & $9.73 \pm 0.83$ & $4.08 \pm 0.63$ & $4.00 \pm 0.69$ & $7.08 \pm 0.88$ & $6.24 \pm 0.69$ & $F_{(4,72)}=11.97, p<0.001$, partialeta $^{2}=0.4$ \\
\hline Stage 2 (min) & $44.37 \pm 3.7$ & $40.13 \pm 3.30$ & $46.28 \pm 4.3$ & $44.89 \pm 2.6$ & $44.10 \pm 2.9$ & $F_{(4,72)}=0.57, p<0.67$, partialeta $^{2}=0.03$ \\
\hline SWS (min) & $9.00 \pm 2.7$ & $23.07 \pm 3.5$ & $23.03 \pm 4.9$ & $18.87 \pm 3.6$ & $22.37 \pm 4.1$ & $F_{(4,72)}=4.36, p=0.003$, partialeta $^{2}=0.19$ \\
\hline REM (min) & $23.71 \pm 3.2$ & $24.05 \pm 2.9$ & $18.21 \pm 2.5$ & $14.61 \pm 1.9$ & $23.13 \pm 2.9$ & $F_{(4,72)}=2.68, p=0.03$, partialeta $^{2}=0.13$ \\
\hline
\end{tabular}

Table 4. Study 2: experimental nap (polysomnography)

\begin{tabular}{|c|c|c|c|c|}
\hline & Placebo & Sodium oxybate $(2.5 \mathrm{~g})$ & Zolpidem (10 mg) & Statistic \\
\hline $\mathrm{TST}^{\text {nap }}(\mathrm{min})$ & $92 \pm 3.2$ & $91 \pm 2.3$ & $93 \pm 1.3$ & $F_{(2,58)}=0.08, p=0.91$, partial eta ${ }^{2}=0.003$ \\
\hline Sleep latency ${ }^{\text {nap }}$ (min) & $9.2 \pm 1.2$ & $8.8 \pm 0.94$ & $10.5 \pm 1.3$ & $F_{(2,58)}=0.65, p=0.52$, partial eta ${ }^{2}=0.02$ \\
\hline WASO $^{\text {nap }}(\mathrm{min})$ & $17.7 \pm 3.8$ & $13.7 \pm 2.4$ & $13.8 \pm 2.7$ & $F_{(2,58)}=0.08, p=0.91$, partial eta ${ }^{2}=0.003$ \\
\hline Sleep efficiency ${ }^{\text {nap }}$ & $78.9 \pm 3.1$ & $80.8 \pm 2.2$ & $80.5 \pm 1.8$ & $F_{(2,58)}=0.14, p=0.86$, partial eta ${ }^{2}=0.005$ \\
\hline Stage 1 (min) & $8.3 \pm 0.87$ & $6.1 \pm 0.68$ & $7.88 \pm 0.95$ & $F_{(2,58)}=2.26, p=0.11$, partial eta ${ }^{2}=0.07$ \\
\hline Stage 2 (min) & $43.6 \pm 2.6$ & $40.47 \pm 2.88$ & $39.2 \pm 3.0$ & $F_{(2,58)}=1.0, p=0.34$, partial eta ${ }^{2}=0.03$ \\
\hline SWS (min) & $9.8 \pm 1.5$ & $19.26 \pm 3.0$ & $24.3 \pm 2.0$ & $F_{(2,58)}=10.6, p<0.001$, partial eta ${ }^{2}=0.26$ \\
\hline REM (min) & $30.7 \pm 2.3$ & $25.9 \pm 2.4$ & $22.6 \pm 2.4$ & $F_{(2,58)}=4.55, p=0.01$, partial eta ${ }^{2}=0.13$ \\
\hline
\end{tabular}

Table 5. Study 2: experimental nap (spindle analysis)

\begin{tabular}{|c|c|c|c|c|c|}
\hline Spindle feature & Channel & Placebo & Sodium oxybate $(2.5 \mathrm{~g})$ & Zolpidem (10 mg) & Statistic \\
\hline \multirow[t]{2}{*}{ Density } & $C 4$ & $2.40 \pm 0.14$ & $1.68 \pm 0.21$ & $3.13 \pm 0.24$ & $F_{(2,58)}=19.64, p<0.001$, partial eta ${ }^{2}=0.40$ \\
\hline & C3 & $2.78 \pm 0.17$ & $2.03 \pm 0.25$ & $3.45 \pm 0.27$ & $F_{(2.58)}=12.45, p<0.001$, partial eta ${ }^{2}=0.30$ \\
\hline \multirow[t]{2}{*}{ Amplitude } & C4 & $16.96 \pm 0.45$ & $17.31 \pm 0.46$ & $16.43 \pm 0.48$ & $F_{(2,56)}=3.75, p=0.03$, partial eta $^{2}=0.11$ \\
\hline & $\mathrm{C} 3$ & $16.78 \pm 0.52$ & $17.01 \pm 0.49$ & $15.63 \pm 0.44$ & $F_{(2,56)}=7.39, p=0.001$, partial eta $^{2}=0.17$ \\
\hline \multirow[t]{2}{*}{ Frequency } & $C 4$ & $13.58 \pm 0.09$ & $13.49 \pm 0.09$ & $13.49 \pm 0.08$ & $F_{(2,50)}=10.81, p<0.001$, partial eta ${ }^{2}=0.20$ \\
\hline & C 3 & $13.49 \pm 0.10$ & $13.48 \pm 0.09$ & $13.43 \pm 0.09$ & $F_{(2,56)}=1.33, p=0.27$, partial eta $^{2}=0.04$ \\
\hline \multirow[t]{2}{*}{ Fast spindle density } & $C 4$ & $1.59 \pm 0.23$ & $1.23 \pm 0.22$ & $2.23 \pm 0.27$ & $F_{(2,52)}=10.81, p<0.001$, partial eta ${ }^{2}=0.29$ \\
\hline & $\mathrm{C} 3$ & $1.85 \pm 0.29$ & $1.45 \pm 0.25$ & $2.40 \pm 0.30$ & $F_{(2,54)}=7.35, p=0.002$, partial eta $^{2}=0.21$ \\
\hline \multirow[t]{2}{*}{ Slow spindle density } & C4 & $0.77 \pm 0.13$ & $0.64 \pm 0.11$ & $1.46 \pm 0.26$ & $F_{(2,56)}=9.49, p<0.001$, partial eta ${ }^{2}=0.25$ \\
\hline & $\mathrm{C} 3$ & $1.18 \pm 0.22$ & $0.80 \pm 0.13$ & $1.84 \pm 0.39$ & $F_{(2,52)}=5.46, p=0.007$, partial eta $^{2}=0.17$ \\
\hline \multirow[t]{2}{*}{ Fast spindle amplitude } & C4 & $16.34 \pm 0.50$ & $16.77 \pm 0.48$ & $15.88 \pm 0.56$ & $F_{(2,50)}=2.49, p=0.09$, partial eta $^{2}=0.09$ \\
\hline & C 3 & $16.49 \pm 0.68$ & $16.32 \pm 0.55$ & $15.16 \pm 0.50$ & $F_{(2,54)}=2.74, p=0.07$, partial eta ${ }^{2}=0.09$ \\
\hline \multirow[t]{2}{*}{ Slow spindle amplitude } & C4 & $17.20 \pm 0.52$ & $17.30 \pm 0.43$ & $16.66 \pm 0.50$ & $F_{(2,56)}=1.84, p=0.168$, partial eta $^{2}=0.06$ \\
\hline & $\mathrm{C} 3$ & $16.94 \pm 0.52$ & $17.28 \pm 0.48$ & $15.64 \pm 0.42$ & $F_{(2,56)}=10.36, p<0.001$, partial eta ${ }^{2}=0.27$ \\
\hline
\end{tabular}

combined amplitude (i.e., average of fast and slow spindles) across hemispheres and found no significant difference $(p=$ 0.245 ) across conditions. We present C4 only in the drug/behavioral interactions for fast, slow, and combined spindles, as the outcome for C3 was comparable (Table 5). Significant differences were found in comparisons of fast $(p<0.001)$ and slow $(p<$ $0.001)$ spindle densities separately. ZOL produced more slow $(p=0.007)$ and fast $(p=0.012)$ spindles than placebo, whereas SO produced fewer fast $(p=0.008)$ and marginally fewer slow ( $p=0.077)$ spindles than placebo. No differences were found between conditions in spindle frequency $(p=0.229)$ and amplitude of fast ( $p=0.093)$ or slow $(p=0.168)$ spindles. Combined spindle amplitudes varied across conditions $(p=0.030)$; however, there were no significant differences between ZOL and placebo $(p=0.104)$ or SO and placebo $(p=0.330)$.

For total slow oscillation power $(0.5-1.0 \mathrm{~Hz})$ in NREM sleep, no differences were found across drug conditions in the left hemisphere $\left(\mathrm{C} 3: F_{(2,58)}=2.17, p=0.12\right)$ or the right hemisphere (C4: $\left.F_{(2,58)}=1.47, p=0.23\right)$.

\section{Increased spindles improve hippocampal memory}

Before examining the effect of drug condition on memory consolidation, we ruled out an encoding effect by testing for baseline differences across drug conditions in the encoding sessions: texture discrimination $(p=0.87)$, word-pair associates $(p=0.10)$, and motor sequence task $(p=0.15)$. Next, we analyzed the effects of drug condition on memory consolidation for each task by running a repeated-measures ANOVA on the difference score between encoding and test sessions [for the verbal task, immediate (A.M.) and delayed (P.M.) test] across the three drug conditions (Fig. 3, Table 6).

For the verbal task, a significant difference was found in performance across drug conditions $(p=0.004)$ (Fig. 4a). Verbal memory improvement was observed in the ZOL conditions ( $p=$ 0.03 ) and not the SO condition $(p=0.21)$ relative to placebo. These memory performance differences were in agreement with our hypothesis, except for the fact that SO, though numerically lower than placebo, did not produce significant differences in the ANOVA. To further test our hypothesis that there would be a 


\section{Verbal Paired Associates}

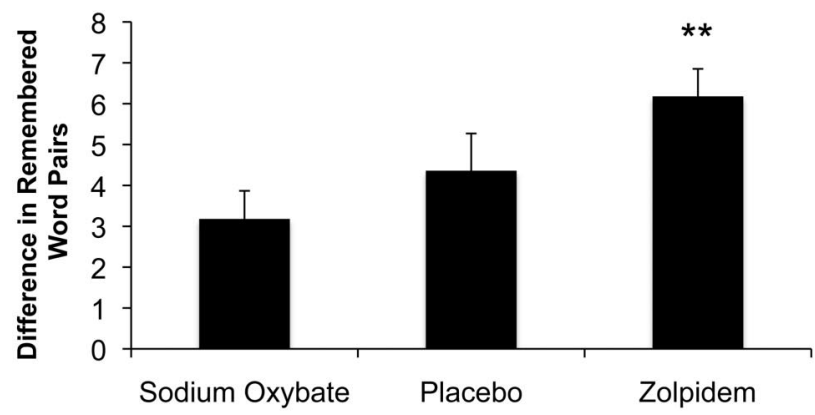

Visual Texture Discrimination

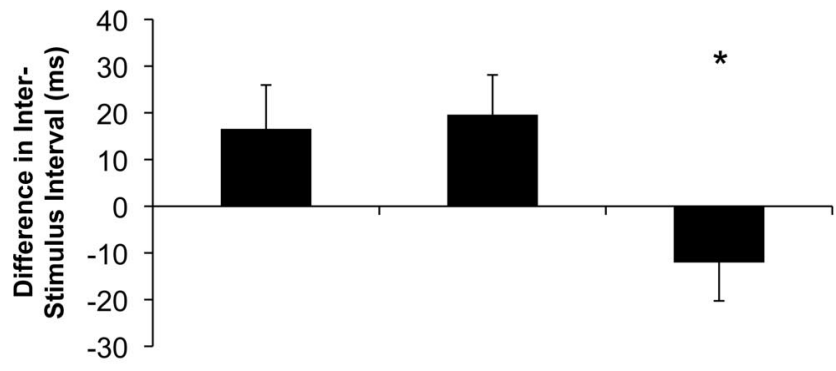

Sodium Oxybate Placebo Zolpidem

\section{Motor Sequence Learning}

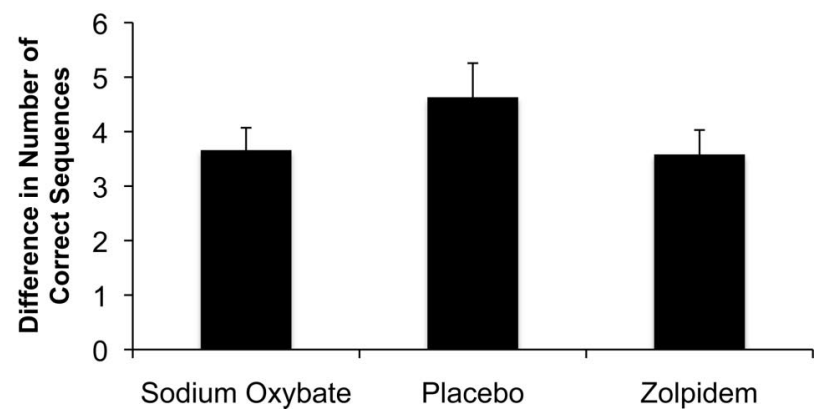

Figure 3. Cognitive performance by drug condition. Difference scores for the verbal paired associate task, the visual texture discrimination task, and the motor sequence task for the three drug conditions: $10 \mathrm{mg}$ of $\mathrm{ZOL}, 2.5 \mathrm{~g}$ of S0, and placebo. Statistical comparisons are between drug conditions and placebo. ${ }^{* *} p<0.005 ;{ }^{*} p<0.05$.

linear trend in memory performance (i.e., $\mathrm{SO}<$ placebo $<\mathrm{ZOL}$ ), we ran a planned contrast analysis that compared the difference scores in SO, placebo, and ZOL with contrast weights $(-1,0,1)$ respectively. Here, our hypothesis significantly predicted the data $\left(t_{(28)}=3.89 ; p=0.0002\right.$, one-tailed $)$. There was a difference across drug conditions in perceptual learning $(p=0.02)$ and post hoc $t$ tests showed significantly decreased learning in ZOL ( $p=$ $0.01)$ and no differences between SO $(p=0.77)$ compared with placebo (Fig. 4b). Contrary to our predictions, we found no significant differences across drug conditions on the motor sequence task $(p=0.65)$ (Fig. $4 c)$, which underscores the specificity of these effects.

Correlations were run between performance on the three memory tasks and nap sleep features (TST, SL, WASO, SE, and minutes of Stage 1, Stage 2, SWS, and REM sleep) in each of the drug conditions. In the ZOL condition, no significant correlations with these sleep features were found for the motor task $(r<$ $0.25, p>0.20$ ), verbal task [all correlations were $r<0.013, p>$
0.26 , except Stage $1 \mathrm{~min} r=-0.30, p=0.11$ ), or perceptual task $(r<0.12, p>0.20)$. Similarly, no significant correlations were found in placebo condition for the motor task $(r<0.14, p>$ $0.44)$, verbal task [all correlations were $r<0.12, p>0.50$, except REM minutes $(r=-0.15, p=0.16)$ ], and perceptual task [all correlations were $r<0.15, p>0.20$, except SL $(r=-0.15, p=$ $0.15)$ and SE $(r=0.14, p=0.18)]$. Finally, SO also did not show any significant correlations between sleep features and performance for the motor task $(r<0.12, p>0.38)$, verbal task [all correlations were $r<0.25, p>0.20$, except Stage $2 \min (r=0.32$ $p=0.08)$ ], and perceptual task [all correlations were $r<0.21$, $p>0.23$, except SE $(r=0.31, p=0.09), \mathrm{SL}(r=-0.32, p=0.09)$, and WASO $(r=-0.28, p=0.14)]$.

We next examined whether the drug effects on memory could be related to postnap differences in sleepiness with a repeatedmeasures ANOVA between sleepiness (KSS scores at 6:00 A.M., 7:00 A.M., 10:30 A.M., 3:00 P.M., and 4:00 P.M.) by drug condition. The sleepiness-by-condition interaction term was not significant $(p=0.53)$, indicating that sleepiness was modulated similarly throughout the experimental day across all conditions. We further probed the potential confound of sleepiness on performance by examining whether the change in sleepiness from prenap to postnap was correlated with the change in performance and again found no significant correlation in any drug condition $(p>0.28)$. Thus, variation in sleepiness or alertness attributable to drug condition did not appear to play an important role in the pharmacologically enhanced learning.

We next examined the relationship between Stage 2 spindle density and the magnitude of verbal memory improvement in the three drug conditions. Verbal memory performance was significantly correlated with spindle density in ZOL $(r=0.38, p=0.02)$ and placebo $(r=0.34, p=0.02)$ but was not significant in SO $(r=0.29, p=0.08)$. Importantly, no significant correlations were found between spindle density and either texture discrimination (where ZOL decreased performance) or motor learning (where no performance changes were observed) in any drug condition (except see correlations between performance and fast and slow spindles below).

Previous studies have discussed the importance of slow wave oscillations for declarative memory consolidation (Molle et al., 2002; Marshall et al., 2006). Therefore, we examined the association between total slow oscillation power in NREM sleep and performance on the three tasks in each drug condition. In the placebo condition, performance was not significantly correlated with slow oscillations in the motor task (C3: $r=-0.16, p=0.39$; or C4: $r=-0.19, p=0.30)$, perceptual task (C3: $r=-0.10$, $p=0.60$; or C4: $r=0.11, p=0.54)$, or verbal task (C3: $r=0.13$, $p=0.49$; or $\mathrm{C} 4: r=0.22, p=0.24)$. Similarly, no significant correlations were found in the SO condition with performance for the motor task (C3: $r=-0.11, p=0.55$; or C4: $r=-0.14$, $p=0.43)$, perceptual task (C3: $r=0.05, p=0.77$; or C4: $r=$ $0.13, p=0.49)$, and verbal task (C3: $r=0.04, p=0.80$; or C4: $r=$ $0.03, p=0.84)$. Finally, in the ZOL condition, no significant correlations were found in the motor task $(\mathrm{C} 3: r=0.12, p=0.52$; or C4: $r=0.16, p=0.38)$, perceptual task $(\mathrm{C} 3: r=0.06, p=0.73$; or C4: $r=-0.22, p=0.25$ ), or verbal task (note the nonsignificant effect in the right hemisphere) (C3: $r=-0.14, p=0.45$; or C4: $r=-0.32, p=0.08$ ).

To further probe the relationship between Stage 2 spindle density and enhancements in verbal memory that was independent of the drug conditions, we used ANCOVA with verbal memory performance as the dependent variable, drug condition as the independent variable, and spindle density as the covariate. The 
Table 6. Study 2: task performance for encoding and test sessions

\begin{tabular}{|c|c|c|c|c|c|c|}
\hline & \multicolumn{2}{|l|}{ Placebo } & \multicolumn{2}{|c|}{ Sodium oxybate $(2.5 \mathrm{~g})$} & \multicolumn{2}{|c|}{ Zolpidem (10 mg) } \\
\hline & $\overline{\text { A.M. }}$ & P.M. & $\overline{\text { A.M. }}$ & P.M. & $\overline{\text { A.M. }}$ & P.M. \\
\hline $\begin{array}{l}\text { Verbal memory: word-pair associates (number } \\
\text { of correct pairs recalled out of } 48 \text { pairs) }\end{array}$ & $24.5 \pm 1.5$ & $28.9 \pm 1.3$ & $26.7 \pm 1.2$ & $30.1 \pm 1.0$ & $24.1 \pm 1.5$ & $30.4 \pm 1.0^{*}$ \\
\hline $\begin{array}{l}\text { Perceptual learning: texture discrimination } \\
\text { (threshold in ms) }\end{array}$ & $153.1 \pm 16.9$ & $133.4 \pm 13.0$ & $149.6 \pm 16.4$ & $132.1 \pm 13.0$ & $151.3 \pm 17.0$ & $163.4 \pm 17.0^{*}$ \\
\hline $\begin{array}{l}\text { Motor learning: motor sequence (number of } \\
\text { correct sequences) }\end{array}$ & $20.0 \pm 0.97$ & $24.7 \pm 1.0$ & $21.5 \pm 1.0$ & $25.2 \pm 1.1$ & $20.8 \pm 0.83$ & $24.4 \pm 0.91$ \\
\hline
\end{tabular}
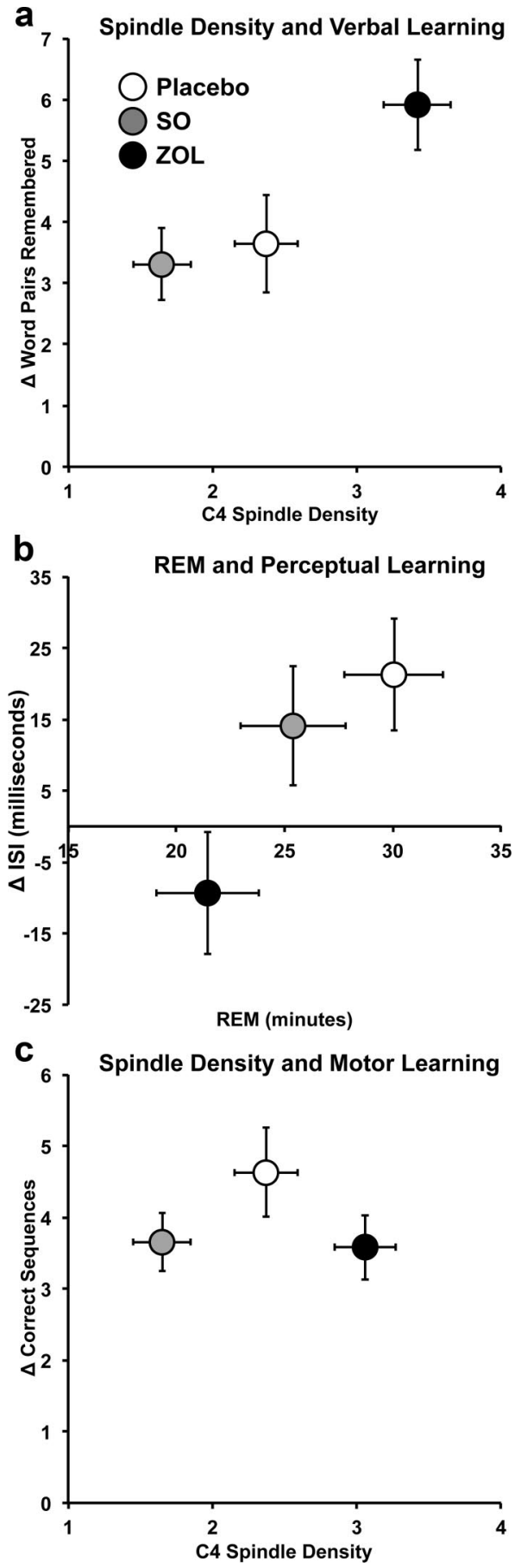

Figure 4. Sleep features and memory performance. $\boldsymbol{a}$, Spindle density in each drug condition (ZOL, black circle; SO, gray circle; placebo, white circle) is shown relative to change scores in verbal memory. $\boldsymbol{b}$, REM minutes in each drug are shown relative to change scores in perceptual learning. $\boldsymbol{c}$, Spindle density in each drug condition is shown relative to change scores in motor learning.
ANCOVA showed that when spindle density is accounted for, the main effect of drug condition disappears $(p=0.40)$, whereas the spindle density effect on performance was highly significant $(p<0.001)$.

Together, these findings indicate that the verbal memory improvements with ZOL seem to represent an enhancement of a normal consolidation process during sleep, as similar correlations between spindles and verbal memory were found in all three drug conditions and there were no detectable differences between spindles in amplitude or frequency between ZOL and placebo. In addition, the experimental manipulation of spindles and associated increases in verbal memory raise the possibility that sleep spindles may represent physiological processes critical for hippocampal-dependent verbal memory consolidation, evinced by the significant effect of spindles on performance in the ANCOVA. Importantly, we did not find improved verbal memory to be related to any other sleep features (e.g., sleep duration, sleep efficiency, etc.) whether in the placebo condition or in changes associated with either of the active drugs.

To further dissect the effects of fast and slow spindles on memory performance, we correlated fast and slow spindle density, amplitude, and average frequency within each drug condition with difference scores on the verbal, perceptual, and motor tasks. For verbal memory, only overall spindle density was significantly correlated with performance in the three drug conditions.

For perceptual learning, however, improvements under the placebo condition were negatively correlated with slow spindle density $(r=-0.41, p=0.02)$, and positively correlated with fast spindle frequency $(r=0.50, p=0.006)$ and amplitude $(r=0.47$, $p=0.01)$. SO perceptual learning was also negatively correlated with slow spindle density $(r=-0.50, p=0.006)$, but not with any other spindle feature $(p>0.25)$, and no ZOL spindle features were significantly correlated with perceptual learning $(p>0.44)$.

For motor memory, negative correlations with slow spindle density were found in SO $(r=-0.38, p=0.03)$, but no other spindle feature was correlated $(p>0.85)$. A marginally significant positive correlation was found between placebo motor learning and fast spindle density $(r=0.35, p=0.05)$, whereas no correlations were found in ZOL ( $p>0.45)$. In summary, perceptual learning was negatively correlated with fast spindle characteristics in the placebo and SO conditions, and a weak association with fast spindle density emerged in both the placebo and SO conditions for motor learning; however, the improvements found in verbal memory did not seem to distinguish fast or slow spindles.

\section{Discussion}

Through pharmacological intervention during sleep, we demonstrate a causal increase in hippocampal-dependent verbal memory performance and suggest that sleep spindles may mediate this enhancement. More specifically, we show that phar- 
macologically enhancing sleep spindles in healthy adults produces exceptional memory performance beyond that seen with sleep alone (placebo) or sleep with the comparison drug (SO). The hippocampus has previously been shown to particularly engage learning of associative elements (Davachi and Wagner, 2002; Yoon et al., 2012), and associative verbal learning has been correlated with sleep spindles (Gais et al., 2002; Schabus et al., 2004; Clemens et al., 2005; Schmidt et al., 2006). Therefore, we used an associative verbal learning task to test the prediction that sleep spindles play a critical role in hippocampal-dependent memory consolidation.

The main finding of our study, that spindles are critical for hippocampal-dependent verbal memory, is supported by the following results. First, only the density of spindles in the ZOL condition differed from placebo, whereas by other measures (i.e., amplitude and frequency of fast, slow, and combined spindles) ZOL spindles appeared similar to the placebo condition. Other studies have noted that pharmacological manipulation of sleep can produce spindles that differ from spontaneous spindles in several ways (Mackenzie et al., 2004; Ferenets et al., 2006). However, the similarity of spindles across drug conditions in the present study is key in our identification of a common mechanism with the same functional consequences as normal sleep. Second, although neurophysiological conditions between daytime and nighttime sleep differ, it is unlikely that the performance differences were caused by circadian influences or other sleep confounds as the nap paradigm avoids time-of-day differences between conditions and prior sleep was carefully controlled throughout the experiment using actigraphy, sleep diaries, and PSG. Third, the results are unlikely attributable to side effects of the experimental hypnotics. Neither sleepiness nor subjective drug effects correlated significantly with changes in memory performance. Finally, total sleep time did not correlate with performance on the three memory tasks, consistent with prior studies showing consolidation is related to sleep quality and not sleep quantity (Mednick et al., 2003; Cai et al., 2009).

Since total sleep time in the naps was held constant across conditions, these high spindle density ZOL naps also showed reduced time in REM sleep that led to a concomitant decrease in perceptual learning. A similar tradeoff between REM sleep and spindle activity has been shown with pharmacological suppression of REM sleep using selective serotonin and norepinephrine reuptake inhibitors (SSRI and SNRI, respectively) (Rasch et al., 2009). In this study, reduced REM increased motor sequence learning and was correlated with the increase in density of fast spindles between placebo and drug conditions. In the present study, we found little effect of spindle density modulation on the motor sequence learning task, except for a correlation with fast spindle density in the placebo condition that replicated previous results (Rasch et al., 2009; Barakat et al., 2011). We also did not find changes in slow oscillation power caused by drug condition or significant correlations between slow oscillation power and verbal memory performance, as others have shown (Marshall et al., 2006). The lack of an association may be related to the fact that although ZOL increased SWS, slow oscillations were not specifically enhanced. Furthermore, the majority of studies examining slow oscillation power have been in nighttime sleep, which may produce different results from early morning naps because of increased sleep pressure and duration.

Contrary to our predictions, motor learning was not altered by the drug intervention and performance was not correlated with spindles in the ZOL condition. A similar null result in motor learning was found by enhancing slow wave activity and spindles via transcranial application of slow oscillations $(0.75 \mathrm{~Hz})$ (Marshall et al., 2006). Although the increase in slow wave activity produced improvement in word-pair associate learning, motor sequence learning did not change. Together, these results indicate a tighter coupling between sleep spindles and declarative verbal memory than motor learning. This difference may be attributable to the stronger dependence on hippocampal processing in the verbal task because of the associative nature of the task (Henke et al., 1999; Davachi and Wagner, 2002). In the case of hippocampal-dependent processing, spindles may be causally related to memory improvement by their direct role in hippocampal replay, whereas in nonhippocampal-dependent tasks, spindles may be a marker of a yet undefined process that correlates with consolidation.

Although enhancing sleep spindle density pharmacologically had a positive effect on verbal memory, we only found a small decrease in verbal memory associated with decreasing spindles in the SO condition. One possibility is that the magnitude of the decrease was too low to have a massively deleterious effect on declarative memory, as spindles were not totally eliminated. A formal test of this hypothesis would be to experimentally inhibit most or all spindles and examine the resultant memory outcomes associated with this elimination (e.g., perhaps with a higher dose of $\mathrm{SO}$ ). Normal aging provides a natural experiment in which spindles show significant reduction in number and density compared with younger adults (Nicolas et al., 2001; Crowley et al., 2002; Rauchs et al., 2008). However, few studies have examined the precise relationship between the decline in spindles and well documented impairments in hippocampal-related memory tasks such as word-pair associate learning (Buckner, 2004; Hornung et al., 2005). Investigation into pharmacologically increasing sleep spindles in the elderly as a declarative memory enhancer may be a promising direction for future cognitive therapy (Pace-Schott and Spencer, 2011).

What might be the mechanism for this exceptional verbal memory enhancement by zolpidem? In other words, what might be the critical component of zolpidem's action that creates the ideal conditions for the transfer of recent memories from the hippocampus to cortical long-term memory stores (i.e., neural replay)? Several investigators have proposed that replay is performed by coordinated rhythmic patterns of sharp wave ripple complexes in the hippocampus and thalamocortical spindles (Buzsáki, 1989, 1996; McClelland et al., 1995). Consistent with this, zolpidem increases two EEG waveforms associated with replay: spindles (shown here) and hippocampal sharp wave ripple complexes (Koniaris et al., 2011). Therefore, it is interesting to consider whether the neural state promoted by zolpidem is an example of the condition that promotes replay-guided, hippocampal memory consolidation.

A curious aspect of zolpidem is the purported amnestic effect of the drug for information experienced after administration (i.e., anterograde amnesia) in humans (Wesensten et al., 1995; Troy et al., 2000) and in animals (Berlin et al., 1993). Along with increased anterograde amnesia, zolpidem reduces synaptic plasticity (Higashima et al., 1998) and facilitates the maintenance of previously induced memories (Cai, 2010). An important and unanswered question is whether the reduction in the ability to form new memories is a necessary foundation for replay-driven, declarative memory consolidation, or is this merely an epiphenomenal aspect of the drug? The answer to this question will greatly inform our understanding of the exact mechanism underlying declarative memory consolidation. Although not directly tested in the present study, we hypothesize that the inhibition of new 
encoding (i.e., reduced synaptic plasticity and increased anterograde amnesia) may be a critical precondition for hippocampaldependent memory consolidation (Mednick et al., 2011). From this general principle, we extrapolate that, more than NREM sleep, zolpidem may enhance consolidation of recently learned memories by strongly inhibiting the formation of newer memories. Further research into the role of plasticity during sleep is needed to help shape future computational and psychological models of memory consolidation. In conclusion, these findings establish a causal link between pharmacological intervention during sleep and the consolidation of a discrete memory domain and implicate sleep spindles as a possible mechanism. The results set the stage for targeted treatment of memory impairments as well as the possibility of exceptional memory improvement above that of a normal sleep period.

\section{References}

Andriamampandry C, Taleb O, Kemmel V, Humbert JP, Aunis D, Maitre M (2007) Cloning and functional characterization of a gammahydroxybutyrate receptor identified in the human brain. FASEB J 21: 885-895. CrossRef Medline

Barakat M, Doyon J, Debas K, Vandewalle G, Morin A, Poirier G, Martin N, Lafortune M, Karni A, Ungerleider LG, Benali H, Carrier J (2011) Fast and slow spindle involvement in the consolidation of a new motor sequence. Behav Brain Res 217:117-121. CrossRef Medline

Berlin I, Warot D, Hergueta T, Molinier P, Bagot C, Puech AJ (1993) Comparison of the effects of zolpidem and triazolam on memory functions, psychomotor performances, and postural sway in healthy subjects. J Clin Psychopharmacol 13:100-106. Medline

Brunner DP, Dijk DJ, Munch M, Borbely AA (1991) Effect of zolpidem on sleep and sleep EEG spectra in healthy young men. Psychopharmacology (Berl) 104:1-5. CrossRef Medline

Buckner RL (2004) Memory and executive function in aging and AD: multiple factors that cause decline and reserve factors that compensate. Neuron 44:195-208. CrossRef Medline

Buzsáki G (1989) Two-stage model of memory trace formation: a role for "noisy" brain states. Neuroscience 31:551-570. CrossRef Medline

Buzsáki G (1996) The hippocampo-neocortical dialogue. Cereb Cortex 6:81-92. CrossRef Medline

Cai DJ (2010) Evidence for sleep-dependent memory consolidation in humans and mice. In: Psychology, p 93. La Jolla, CA: University of California, San Diego.

Cai DJ, Mednick SA, Harrison EM, Kanady JC, Mednick SC (2009) REM, not incubation, improves creativity by priming associative networks. Proc Natl Acad Sci U S A 106:10130-10134. Medline

Clemens Z, Fabó D, Halász P (2005) Overnight verbal memory retention correlates with the number of sleep spindles. Neuroscience 132:529-535. CrossRef Medline

Crowley K, Trinder J, Kim Y, Carrington M, Colrain IM (2002) The effects of normal aging on sleep spindle and $\mathrm{K}$-complex production. Clin Neurophysiol 113:1615-1622. CrossRef Medline

Davachi L, Wagner AD (2002) Hippocampal contributions to episodic encoding: insights from relational and item-based learning. J Neurophysiol 88:982-990. Medline

De Gennaro L, Ferrara M (2003) Sleep spindles: an overview. Sleep Med Rev 7:423-440. CrossRef Medline

Diekelmann S, Born J (2010) The memory function of sleep. Nat Rev Neurosci 11:114-126. CrossRef Medline

Drover DR (2004) Comparative pharmacokinetics and pharmacodynamics of short-acting hypnosedatives: zaleplon, zolpidem and zopiclone. Clin Pharmacokinet 43:227-238. CrossRef Medline

Eschenko O, Mölle M, Born J, Sara SJ (2006) Elevated sleep spindle density after learning or after retrieval in rats. J Neurosci 26:12914-12920. CrossRef Medline

Farrant M, Nusser Z (2005) Variations on an inhibitory theme: phasic and tonic activation of GABA(A) receptors. Nat Rev Neurosci 6:215-229. CrossRef Medline

Feinberg I, Maloney T, Campbell IG (2000) Effects of hypnotics on the sleep EEG of healthy young adults: new data and psychopharmacologic implications. J Psychiatr Res 34:423-438. Medline
Ferenets R, Lipping T, Suominen P, Turunen J, Puumala P, JänttiV, Himanen SL, Huotari AM (2006) Comparison of the properties of EEG spindles in sleep and propofol anesthesia. Conf Proc IEEE Eng Med Biol Soc 1:63566359. CrossRef Medline

Fogel SM, Smith CT, Beninger RJ (2009) Evidence for 2-stage models of sleep and memory: learning-dependent changes in spindles and theta in rats. Brain Res Bull 79:445-451. CrossRef Medline

Gais S, Mölle M, Helms K, Born J (2002) Learning-dependent increases in sleep spindle density. J Neurosci 22:6830-6834. Medline

Henke K, Weber B, Kneifel S, Wieser HG, Buck A (1999) Human hippocampus associates information in memory. Proc Natl Acad Sci U S A 96:5884-5889. Medline

Higashima M, Kinoshita H, Koshino Y (1998) Differences in the effects of zolpidem and diazepam on recurrent inhibition and long-term potentiation in rat hippocampal slices. Neurosci Lett 245:77-80. CrossRef Medline

Hornung OP, Danker-Hopfe H, Heuser I (2005) Age-related changes in sleep and memory: commonalities and interrelationships. Exp Gerontol 40:279-285. CrossRef Medline

Kaupmann KCJ, Wellendorph P, Mombereau C, Sansig G, Klebs K, Schmutz M, Froestl W, van der Putten H, Mosbacher J, Brauner-Osborne H, Waldmeier P, Bettler B (2003) Specific gamma-hydroxybutyrate-binding sites but loss of pharmacological effects of gamma-hydroxybutyrate in GABA(B)(1)-deficient mice. Eur J Neurosci 18:2722-2730. 10.1111/ j.1460-9568.2003.03013.x $<$ zpmid $>14656321<$ zpmid $><$ bx $>$

$<$ zrefs\%3B26>Koniaris E, Drimala P, Sotiriou E, Papatheodoropoulos $\mathrm{C}<$ zens $>(2011)<$ zens $>$ Different effects of zolpidem and diazepam on hippocampal sharp wave ripple activity in vitro. Neuroscience 175:224234. CrossRef Medline

Louie K, Wilson MA (2001) Temporally structured replay of awake hippocampal ensemble activity during rapid eye movement sleep. Neuron 29:145-156. CrossRef Medline

Mackenzie L, Pope KJ, Willoughby JO (2004) Physiological and pathological spindling phenomena have similar regional EEG power distributions. Brain Res 1008:92-106. CrossRef Medline

Marshall L, Helgadóttir H, Mölle M, Born J (2006) Boosting slow oscillations during sleep potentiates memory. Nature 444:610-613. CrossRef Medline

Marshall L, Kirov R, Brade J, MölleM, Born J (2011) Transcranial electrical currents to probe EEG brain rhythms and memory consolidation during sleep in humans. PLoS One 6:e16905. CrossRef Medline

McClelland JL, McNaughton BL, O'Reilly RC (1995) Why there are complementary learning systems in the hippocampus and neocortex: insights from the successes and failures of connectionist models of learning and memory. Psychol Rev 102:419-457. CrossRef Medline

Mednick S, Nakayama K, Stickgold R (2003) Sleep-dependent learning: a nap is as good as a night. Nat Neurosci 6:697-698. CrossRef Medline

Mednick SC, Nakayama K, Cantero JL, Atienza M, Levin AA, Pathak N, Stickgold R (2002) The restorative effect of naps on perceptual deterioration. Nat Neurosci 5:677-681. CrossRef Medline

Mednick SC, Arman AC, Boynton GM (2005) The time course and specificity of perceptual deterioration. Proc Natl Acad Sci U S A 102:3881-3885. Medline

Mednick SC, Drummond SP, Boynton GM, Awh E, Serences J (2008a) Sleep-dependent learning and practice-dependent deterioration in an orientation discrimination task. Behav Neurosci 122:267-272. CrossRef Medline

Mednick SC, Drummond SP, Arman AC, Boynton GM (2008b) Perceptual deterioration is reflected in the neural response: $\mathrm{fMRI}$ study of nappers and non-nappers. Perception 37:1086-1097. CrossRef Medline

Mednick SC, Cai DJ, Shuman T, Anagnostaras S, Wixted JT (2011) An opportunistic theory of cellular and systems consolidation. Trends Neurosci 34:504-514. CrossRef Medline

Nicolas A, Petit D, RompréS, Montplaisir J (2001) Sleep spindle characteristics in healthy subjects of different age groups. Clin Neurophysiol 112: 521-527. CrossRef Medline

Mölle M, Marshall L, Gais S, Born J (2002) Grouping of spindle activity during slow oscillations in human non-rapid eye movement sleep. J Neurosci 22:10941-10947. Medline

Nishida M, Walker MP (2007) Daytime naps, motor memory consolidation and regionally specific sleep spindles. PLoS One 2:e341. CrossRef Medline 
Pace-Schott EF, Spencer RM (2011) Age-related changes in the cognitive function of sleep. Prog Brain Res 191:75-89. CrossRef Medline

Rasch B, Pommer J, Diekelmann S, Born J (2009) Pharmacological REM sleep suppression paradoxically improves rather than impairs skill memory. Nat Neurosci 12:396-397. CrossRef Medline

Rauchs G, Schabus M, Parapatics S, Bertran F, Clochon P, Hot P, Denise P, Desgranges B, Eustache F, Gruber G, Anderer P (2008) Is there a link between sleep changes and memory in Alzheimer's disease? Neuroreport 19:1159-1162. CrossRef Medline

Rechtschaffen A, Kales A (1968) A manual of standardized terminology, techniques and scoring system for sleep stages of human subjects. Los Angeles: Brain Information Service, University of California, Los Angeles.

Schabus M, Gruber G, Parapatics S, Sauter C, Klösch G, Anderer P, Klimesch W, Saletu B, Zeitlhofer J (2004) Sleep spindles and their significance for declarative memory consolidation. Sleep 27:1479-1485. Medline

Schabus M, Dang-Vu TT, Albouy G, Balteau E, Boly M, Carrier J, Darsaud A, Degueldre C, Desseilles M, Gais S, Phillips C, Rauchs G, Schnakers C, Sterpenich V, Vandewalle G, Luxen A, Maquet P (2007) Hemodynamic cerebral correlates of sleep spindles during human non-rapid eye movement sleep. Proc Natl Acad Sci U S A 104:13164-13169. Medline

Schmidt C, Peigneux P, Muto V, Schenkel M, Knoblauch V, Münch M, de Quervain DJ, Wirz-Justice A, Cajochen C (2006) Encoding difficulty promotes postlearning changes in sleep spindle activity during napping. J Neurosci 26:8976-8982. CrossRef Medline

Siapas AG, Wilson MA (1998) Coordinated interactions between hippocampal ripples and cortical spindles during slow-wave sleep. Neuron 21:1123-1128. CrossRef Medline
Stickgold R, Whidbee D, Schirmer B, Patel V, Hobson JA (2000) Visual discrimination task improvement: a multi-step process occurring during sleep. J Cogn Neurosci 12:246-254. CrossRef Medline

Tamminen J, Payne JD, Stickgold R, Wamsley EJ, Gaskell MG (2010) Sleep spindle activity is associated with the integration of new memories and existing knowledge. J Neurosci 30:14356-14360. CrossRef Medline

Troy SM, Lucki I, Unruh MA, Cevallos WH, Leister CA, Martin PT, Furlan PM, Mangano R (2000) Comparison of the effects of zaleplon, zolpidem, and triazolam on memory, learning, and psychomotor performance. J Clin Psychopharmacol 20:328-337. CrossRef Medline

Walker MP, Brakefield T, Morgan A, Hobson JA, Stickgold R (2002) Practice with sleep makes perfect: sleep-dependent motor skill learning. Neuron 35:205-211. CrossRef Medline

Walsh JK, Hall-Porter JM, Griffin KS, Dodson ER, Forst EH, Curry DT, Eisenstein RD, Schweitzer PK (2010) Enhancing slow wave sleep with sodium oxybate reduces the behavioral and physiological impact of sleep loss. Sleep 33:1217-1225. Medline

Wamsley EJ, Tucker MA, Shinn AK, Ono KE, McKinley SK, Ely AV, Goff DC, Stickgold R, Manoach DS (2012) Reduced sleep spindles and spindle coherence in schizophrenia: mechanisms of impaired memory consolidation? Biol Psychiatry 71:154-161. CrossRef Medline

Wesensten NJ, Balkin TJ, Belenky GL (1995) Effects of daytime administration of zolpidem versus triazolam on memory. Eur J Clin Pharmacol 48:115-122. CrossRef Medline

Yoon J, Seo Y, Kim J, Lee I (2012) Hippocampus is required for paired associate memory with neither delay nor trial uniqueness. Learn Mem 19:1-8. CrossRef Medline 